\title{
Délos : étude de morphologie urbaine - 2017
}

Philippe Fraisse et Lionel Fadin

\section{OpenEdition \\ Journals}

Édition électronique

URL : http://journals.openedition.org/baefe/1888

DOI : $10.4000 /$ baefe. 1888

ISSN : 2732-687X

Éditeur

ResEFE

\section{Référence électronique}

Philippe Fraisse, Lionel Fadin, « Délos : étude de morphologie urbaine - 2017 » [notice archéologique], Bulletin archéologique des Écoles françaises à l'étranger [En ligne], Grèce, mis en ligne le 23 décembre 2020, consulté le 22 mars 2021. URL : http://journals.openedition.org/baefe/1888 ; DOI : https:// doi.org/10.4000/baefe.1888

Ce document a été généré automatiquement le 22 mars 2021.

\section{cc)}

Le Bulletin archéologique des Écoles françaises à l'étranger est mise à disposition selon les termes de la Licence Creative Commons Attribution - Pas d'Utilisation Commerciale - Pas de Modification 4.0 International. 


\title{
Délos : étude de morphologie urbaine - 2017
}

\author{
Philippe Fraisse et Lionel Fadin
}

\section{NOTE DE L'AUTEUR}

Autorité nationale présente : Éphorie des Cyclades

Numéro de mission : C36

Établissements porteurs de l'opération : EFA

\section{Données scientifiques produites :}

Délos par l'EFA

Web SIG de Délos

1 Les travaux programmés pour l'année 2017 se sont déroulés en deux temps. Sur le terrain, les investigations se sont poursuivies, au cours de la dernière semaine d'août, dans le secteur de l'isthme de la presqu'île de Patinioti, au Nord du Stade (Fig. 1). Elles ont été suivies par une première phase d'interprétation des données recueillies en 2014. 
Fig. 1. La région Nord et la presqu'île de Patinioti, vues du Sud.

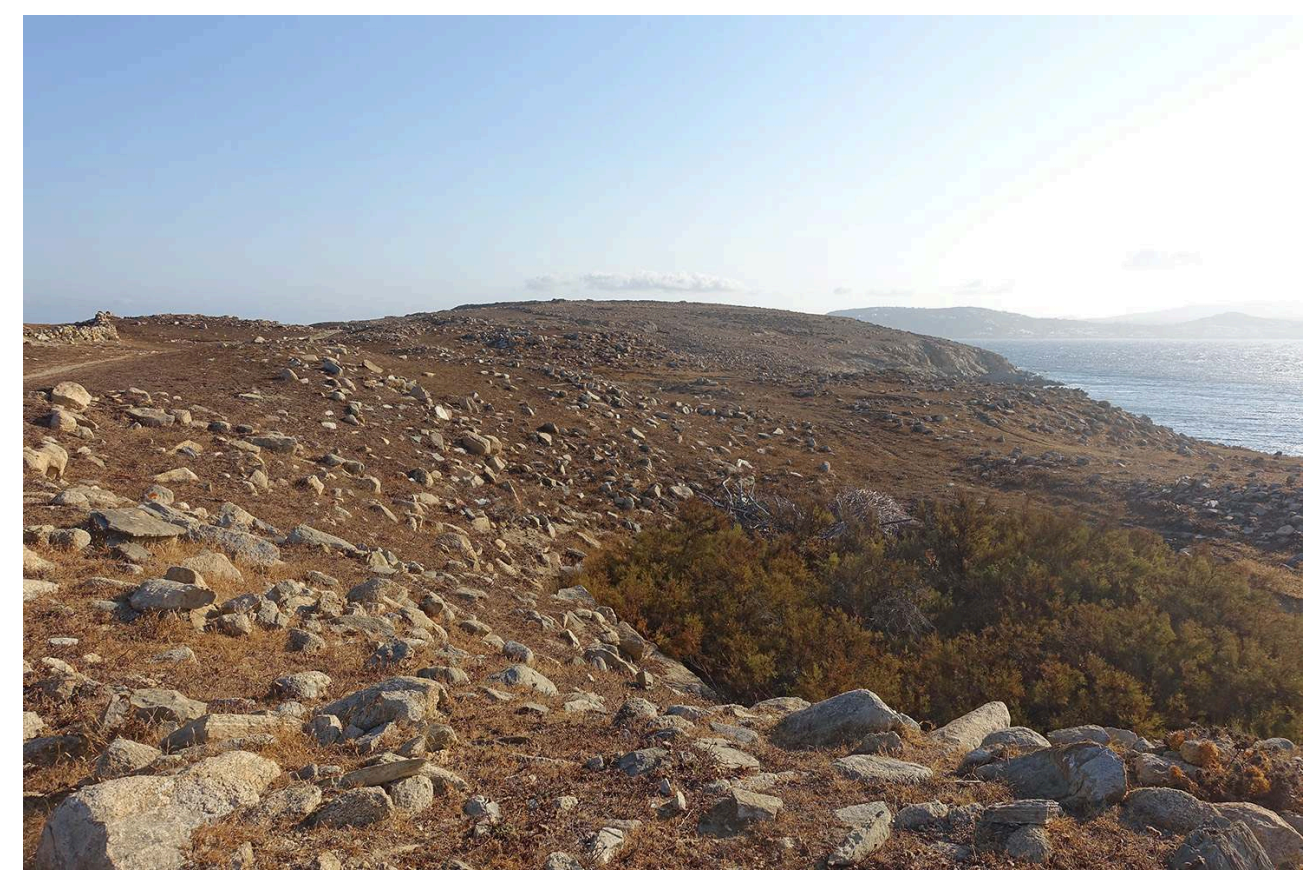

EFA.

2 Les opérations menées sur le terrain avaient pour objectif de compléter l'illustration des structures repérées et relevées en 2014, notamment par le biais de photos aériennes, grâce au survol de cette zone par un drone piloté par Lionel Fadin. Celles-ci n'ont malheureusement pas été facilitées par les conditions climatiques, et notamment par le Meltem qui a soufflé dans la région pratiquement tout le mois d'août. Néanmoins, nous avons pu procéder à 3 vols qui ont permis de réaliser une couverture photographique satisfaisante des structures archéologiques visibles à la surface du terrain naturel.

3 À partir des prises de vues réalisées au cours de cette campagne, il a été possible de construire un orthophotoplan. La superposition de l'image 3D, issue de la couverture photographique, et du plan des vestiges dressé à partir des relevés topographiques de 2014 , constitue un outil d'investigation très utile. L'orthophotoplan permet en effet de mettre en relation directe les structures relevées avec la réalité du terrain. Non seulement il permet de mieux visualiser, sur un fonds photographique en 3D, les structures repérées en surface, mais il offre également la possibilité de repérer d'autres structures bâties, qui restent invisibles à la surface du terrain naturel pour un observateur à pied. Le travail d'interprétation de ces structures, qui est l'objectif de cette phase d'investigation sur le terrain, se trouve dès lors grandement facilité par cette représentation en 3D du secteur.

4 Parallèlement à ces opérations de terrain, plusieurs journées ont été consacrées à la rédaction de deux premiers articles à paraître dans le $\mathrm{BCH}$. Le premier constitue une introduction aux études de morphologie urbaine entreprises sur le site. Il définit le cadre dans lequel cette recherche se déroulera, précisant les éléments de méthode qui devraient permettre d'atteindre les objectifs fixés en vue d'une meilleure connaissance de l'urbanisme à Délos. Le second article constitue une première synthèse qui rassemble les acquis de l'étude conduite sur le secteur de la presqu'île de Patinioti, au 
Nord du Stade. Elle se fonde sur les relevés réalisés en 2014, et cherche à mettre en évidence les logiques qui ont présidées à l'urbanisation d'une région dont la particularité est d'être située, au Sud et au Nord, au contact de la campagne délienne, mais aussi, d'être bordée par la mer sur ses côtes Est et Ouest.

5 L'objectif premier de cette étude était d'acquérir une meilleure compréhension des phénomènes d'urbanisation à l'œuvre dans un secteur qui en réalité constitue le prolongement du Quartier du Stade, fouillé par A. Plassart en 1912. La mise en évidence des règles d'implantation du bâti est au cœur de cette recherche. Elle se fonde sur un travail de géométrie qui vise à révéler les systèmes d'orientations des murs des maisons, en recherchant des correspondances dans les orientations des murs du bâti et des voies constituant la trame urbaine. Un premier traitement des données recueillies a été engagé, à partir duquel ont été élaborées plusieurs hypothèses sur l'organisation du quartier et sur sa trame urbaine.

6 L'interprétation des orientations dominantes du bâti laisse penser que l'on a affaire à deux logiques d'urbanisation distinctes, chacune générant son propre système d'implantation. Un premier plan restitué du quartier a pu être esquissé et constitue l'aboutissement de cette phase.

7 Sur la vaste zone pratiquement horizontale qui prolonge au Nord le Stade, le tissu urbain semble fait d'un certain nombre d'îlots dont la trame suit une orientation NordSud. Le système semble régulier et similaire à ce que l'on observe dans le Quartier du Stade. Une voie principale de direction Nord-Sud, tracée pratiquement dans le prolongement de la rue du Stade, structure cet ensemble, tandis que plusieurs ruelles perpendiculaires, surtout lisibles à l'Ouest de cette voie, irriguent le tissu urbain, permettant la desserte des maisons situées plus à l'intérieur des îlots.

8 Le long de la côte Est, les maisons sont construites sur la falaise et suivent la ligne du rivage ; la topographie, qui, là, est davantage accidentée, a contraint les constructeurs à réaliser des travaux de génie civil importants, afin de construire de solides murs de soutènements. Implantés le plus souvent parallèlement aux courbes de niveaux, ils délimitent des surfaces constructibles plus faciles à aménager. Ainsi, cet ensemble de constructions semble ne pas suivre d'autre logique que celle imposée par le tracé du rivage.

9 Le plan du quartier restitué à partir des relevés topographiques fait nettement apparaître ces deux systèmes. Les îlots réguliers construits sur le plateau appartiennent très certainement au même ensemble urbain que constitue le Quartier du Stade fouillé par A. Plassart. Quant aux constructions implantées sur la ligne de rivage, elles dérogent totalement aux règles qui ont présidé à l'urbanisation de ce quartier et s'apparentent à celles que l'on trouve ailleurs à Délos, par exemple sur la côte Ouest, au pied des collines de Glastropi ou de Ghamila.

10 La présence de ces deux systèmes oblige à se poser la question de la chronologie qui les lie. En l'absence de tout élément de mobilier datable, l'établissement d'une chronologie absolue reste malheureusement chose impossible. Ceci ne doit cependant pas empêcher de réfléchir à une chronologie relative qui permettrait d'établir la primauté d'un système sur l'autre. C'est là l'un des enjeux futurs de l'étude de ce quartier Nord. 


\section{INDEX}

lieux https://ark.frantiq.fr/ark:/26678/pcrtXvsaqTQa4J

Thèmes : EFA

sujets 26678/pcrtKJVpuP3AET, https://ark.frantiq.fr/ark:/26678/crtKTzSReth5I, https:// ark.frantiq.fr/ark:/26678/pcrtaodMT8j830, https://ark.frantiq.fr/ark:/26678/pcrthT7f96LxBR Année de l'opération : 2017

chronologie https://ark.frantiq.fr/ark:/26678/pcrtNzYn31IIAZ

\section{AUTEURS}

\section{PHILIPPE FRAISSE}

École française d'Athènes

\section{LIONEL FADIN}

École française d'Athènes 